\title{
Broad-spectral-range synchronized flat-top arrayed-waveguide grating applied in a 225- channel cascaded spectrometer
}

\author{
B. I. Akca, ${ }^{1, *}$ C. R. Doerr, ${ }^{2}$ G. Sengo, ${ }^{1}$ K. Wörhoff, ${ }^{1}$ M. Pollnau, ${ }^{1}$ and \\ R. M. de Ridder ${ }^{1}$ \\ ${ }^{1}$ Integrated Optical MicroSystems Group, MESA+ Institute for Nanotechnology, University of Twente, \\ 7500 AE Enschede, The Netherlands \\ ${ }^{2}$ Acacia Communications, Inc., Maynard, MA 01754, USA \\ *B.I.Akca@utwente.nl
}

\begin{abstract}
We present a new synchronized design for flattening the passband of an arrayed-waveguide grating (AWG) over a broad wavelength range of $90 \mathrm{~nm}$. A wavelength-insensitive 3-dB balanced coupler is designed to be used in duplicate in a Mach-Zehnder interferometer (MZI); the phase deviation created by one of the balanced couplers is cancelled by flipping the other coupler around. This MZI is arranged in tandem with the AWG such that the output signal of the MZI is the input signal of the AWG. We demonstrate a 5-channel, 18-nm-spacing AWG with a 0.5-dB bandwidth of $12 \mathrm{~nm}$ over a 90-nm spectral range. A low-loss cascaded AWG system is demonstrated by using the MZI-synchronized flat-top AWG as a primary filter.
\end{abstract}

(C)2012 Optical Society of America

OCIS codes: (080.1238) Array waveguide devices; (230.3120) Integrated optics devices.

\section{References and links}

1. M. K. Smit and C. van Dam, "PHASAR-based WDM-devices: Principles, design and applications," IEEE J. Sel. Top. Quantum Electron. 2, 236-250 (1996).

2. B. I. Akca, V. D. Nguyen, J. Kalkman, N. Ismail, G. Sengo, F. Sun, T. G. van Leeuwen, A. Driessen, M Pollnau, K. Wörhoff, and R. M. de Ridder, "Toward spectral-domain optical coherence tomography on a chip,” IEEE J. Sel. Top. Quantum Electron. 18, 1223-1233 (2012).

3. N. Ismail, L. P. Choo-Smith, K. Wörhoff, A. Driessen, A. C. Baclig, P. J. Caspers, G. J. Puppels, R. M. de Ridder, and M. Pollnau, "Raman spectroscopy with an integrated arrayed-waveguide grating," Opt. Lett. 36, 4629-4631 (2011).

4. N. Ismail, B. I. Akca, F. Sun, K. Wörhoff, R. M. de Ridder, M. Pollnau, and A. Driessen, "Integrated approach to laser delivery and confocal signal detection," Opt. Lett. 35, 2741-2743 (2010).

5. M. R. Amersfoort, J. B. D. Soole, H. P. LeBlanc, N. C. Andreadakis, A. Rajhel, and C. Caneau, "Passband broadening of integrated arrayed waveguide filters using multimode interference couplers," Electron. Lett. 32, 449-451 (1996).

6. K. Okamoto and A. Sugita, "Flat spectral response arrayed-waveguide grating multiplexer with parabolic waveguide horns," Electron. Lett. 32, 1661-1662 (1996).

7. K. Okamoto and H. Yamada, "Arrayed-waveguide grating multiplexer with flat spectral response," Opt. Lett. 20, 43-45 (1995).

8. B. Fondeur, A. L. Sala, H. Yamada, N. Gopinathan, D. Nakamoto, and A. Vaidyanathan, "Ultra wide AWG with hyper-Gaussian profile," IEEE Photon. Technol. Lett. 16, 2628-2630 (2004).

9. C. R. Doerr, R. Pafchek, and L. W. Stulz,, "Integrated band demultiplexer using waveguide grating routers," IEEE Photon. Technol. Lett. 23, 1088-1090 (2003).

10. Y. Inoue, M. Oguma, T. Kitoh, M. Ishii, T. Shibata, Y. Hibino, H. Kawata, and T. Sugie, "Low-crosstalk 4-channel coarse WDM filter using silica-based planar-lightwave-circuit," in Optical Fiber Communication Conference, Vol. 70 of OSA Trends in Optics and Photonics Series (Optical Society of America, 2002), paper TuK6.

11. C. Dragone, "Frequency routing device having a wide and substantially flat passband," U. S. Patent 5412744, May 2 (1995). 
12. C. R. Doerr, L. W. Stulz, R. Pafchek, and S. Shunk, "Compact and low-loss manner of waveguide grating router passband flattening and demonstration in a 64-channel blocker/multiplexer," IEEE Photon. Technol. Lett. 14, 56-58 (2002).

13. C. R. Doerr, R. Pafchek, and L. W. Stulz, "Compact and low-loss integrated box-like passband multiplexer," IEEE Photon. Technol. Lett. 15, 918-920 (2003).

14. B. E. Little and T. E. Murphy, "Design rules for maximally flat wavelength-insensitive optical power dividers using Mach-Zehnder structures,” IEEE Photon. Technol. Lett. 9, 1607-1609 (1997).

15. K. Takada, M. Abe, T. Shibata, and K. Okamoto, "10 GHz-spaced 1010-channel tandem AWG filter consisting of one primary and ten secondary AWGs," IEEE Photon. Technol. Lett. 13, 577-578 (2001).

16. K. Takada, H. Yamada, and K. Okamoto, "320-channel multiplexer consisting of a $100 \mathrm{GHz}$-spaced parent AWG and 10 GHz-spaced subsidiary AWGs,” Electron. Lett. 35, 824-826 (1999).

17. K. Takada, M. Abe, T. Shibata, M. Ishii, Y. Inoue, H. Yamada, Y. Hibino, and K. Okamoto, " 10 GHzspaced 1010-channel AWG filter achieved by tandem connection of primary and secondary AWGs," in Proceeding of European. Conference on Optical Communications (IEEE 2000), pp. PD3-8.

18. K. Takada, M. Abe, T. Shibata, and K. Okamoto, "5 GHz-spaced 4200-channel two-stage tandem demultiplexer for ultra-multi-wavelength light source using supercontinuum generation," Electron. Lett. 38, 572-573 (2005).

19. K. Takada, M. Abe, T. Shibata, and K. Okamoto, "Low-loss 10-GHz-spaced tandem multi/demultiplexer with more than 1000 channels using a $1 \times 5$ interference multi/demultiplexer as a primary filter," IEEE Photon. Technol. Lett. 14, 59-61 (2002).

\section{Introduction}

The arrayed-waveguide grating (AWG) is a powerful wavelength-selective device with high accuracy and stability[1]. It is attractive for use in optical wavelength-division-multiplexed networks but also for application to various other spectroscopic measurements, e.g. optical coherence tomography (OCT) [2] and Raman spectroscopy [3], or for confocal light delivery and collection [4]. There is a strong need for AWGs with low-loss and box-like passbands over a broad spectral range. Such AWGs are also known as band demultiplexers which are very useful in both dense and coarse wavelength-division-multiplexed systems. Several approaches have been proposed to implement AWGs having such a flat-top response [5-8], however most have the drawbacks of increased insertion loss and large device size. Doerr et al. demonstrated a novel broad-band flat-top AWG design consisting of two waveguide grating routers, interconnected by a third one of non-standard design [9]. Despite the impressive device performance, the device size was $88 \times 5.4 \mathrm{~mm}^{2}$ which is rather large for further component integration, e.g. cascaded AWG systems. Inoue et al. demonstrated a 20nm-spacing 4-channel coarse wavelength-division-multiplexing filter which comprises a total of 16 Mach-Zehnder interferometer (MZI) stages [10]. Although the filter had the advantages of low loss, low crosstalk, and a wide passband, the device size was $15 \times 71 \mathrm{~mm}^{2}$.

A very effective method for increasing the passband width without introducing intrinsic loss is the so-called MZI-synchronized AWG in which the closely spaced dual-output waveguides of an asymmetrical MZI together form the input waveguides of the AWG, thus generating an input light spot that shifts with wavelength [11-13], as illustrated in Fig. 1a. The term "synchronized" refers to the free spectral range (FSR) of the MZI being equal to the wavelength-channel spacing of the AWG, so that the input light spot for the AWG makes a full 'sweep' in position along the x-direction for each AWG wavelength channel. This synchronous movement of its input spot compensates the dispersion of the AWG for the wavelength range contained in one wavelength channel, thus imaging all these wavelengths at the same output waveguide. Since the FSR of the MZI is equal to the wavelength-channel spacing of the AWG, the resulting device has a flattened passband for all wavelength channels. The MZI configuration given in Refs. [11-13] was constructed by using a Yjunction coupler as the first coupler and a $2 \times 2$ directional coupler as the second coupler. The drawbacks of this configuration are excess loss and limited bandwidth due to the Y-junction coupler and the directional coupler, respectively.

An alternative for the aforementioned couplers is to take advantage of wavelengthinsensitive 3-dB balanced couplers which can be realized by cascading two conventional couplers in a Mach-Zehnder configuration with a relative phase shift of $2 \theta$ introduced between 
them [14], as shown in Fig. 1b. In this configuration, the purpose of the second coupler is to compensate for deviations introduced by the first one. Such couplers can be designed to yield a maximally flat response with respect to deviations in wavelength, polarization, or uniform fabrication over a broad spectral range, with no excess loss. Therefore, these couplers are very good candidates for broad-spectral-range MZI-synchronized AWGs.

a)

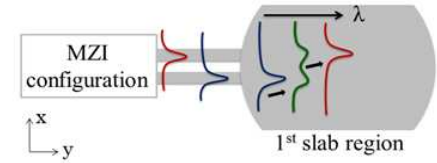

b)

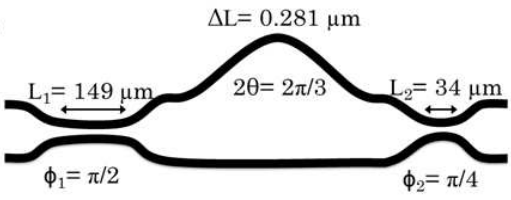

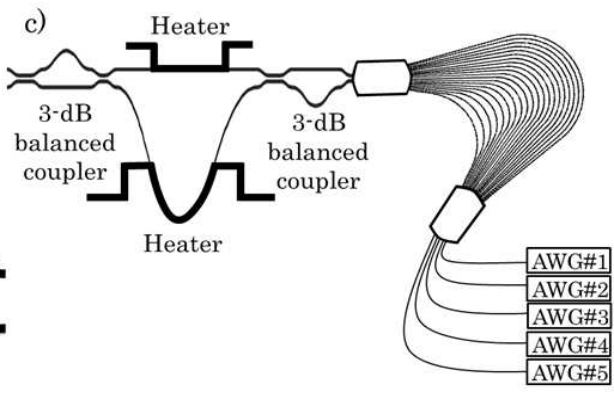

Fig. 1. a) Change in optical field at the MZI and $1^{\text {st }}$ slab region interface of the AWG, as the wavelength is changed. b) Design parameters of the 3-dB balanced coupler. c) Schematic of the cascaded AWG system with a MZI-synchronized AWG using 3-dB balanced couplers. Electrical heaters are placed on both arms of the MZI.

High-density wavelength-division-demultiplexing filters are very important for increasing the capacity of photonic networks. AWG filters can demultiplex very dense wavelengthdivision-multiplexing (WDM) signals; however, it is difficult to combine high resolution and large FSR in a single device, which is highly desirable for numerous applications, e.g. highresolution OCT systems. A cascading configuration enables one to design flexible WDM systems with any channel count and channel spacing [15-18]. Although very-high-density WDM systems have been demonstrated, high loss and small passband due to the Gaussian transfer function of the primary filters are still substantial problems of the cascaded systems. In order to solve these problems flat-passband and low-loss primary AWGs are essential. Takada et al. have demonstrated a partially integrated low-loss multiplexer/demultiplexer configuration by cascading bulky thin-film interference filters as primary filters and five AWGs as secondary filters in order to reduce the overall system loss [19]. Although the loss decreased dramatically, the thin-film interference filters were still not integrated on chip, which is a typical bottleneck for such filters.

In this work we present a new synchronized design for flattening the passband of an AWG over a broad spectral range of $90 \mathrm{~nm}$ without any extra loss. A wavelength-insensitive 3 - $\mathrm{dB}$ balanced coupler is designed to be used in duplicate in the MZI [14]. The phase deviation created by one of the balanced couplers is canceled by flipping the other coupler around. Using these components, a 5-channel, 18-nm-spacing MZI-synchronized AWG is designed and a $0.5-\mathrm{dB}$ bandwidth greater than $12 \mathrm{~nm}$ over $90 \mathrm{~nm}$ spectral range is demonstrated with an overall device size of $11 \times 3 \mathrm{~mm}^{2}$. The relative phase difference between the two MZI arms due to the processing fluctuations is compensated by employing the thermo-optic effect to tune the synchronized MZI. The applicability of this approach is demonstrated by the design and realization of a cascaded AWG system, which consists of this flat-top $1 \times 5$ AWG as a primary filter and five $1 \times 51$ AWGs as secondary filters. Only 45 channels were selected out of 51 channels to resolve the complete spectral content of each primary AWG passband, which resulted in 225 channels in total. The complete device is shown schematically in Fig. 1c. Our configuration is advantageous due to its simple fabrication and full integration.

The FSR and the channel spacing of both, primary and secondary AWGs are chosen arbitrarily in order to prove the feasibility of the proposed design. Generally, these design parameters are flexible for both primary and secondary AWGs. The idea of a broad-band, MZI-synchronized AWG is applicable to high-resolution systems as well. The secondary 
AWGs can be designed with better wavelength resolution, e.g. $0.1 \mathrm{~nm}$, however a compromise between device size and resolution needs to be made.

\section{Design and Simulations}

The splitting ratio at the output of a Mach-Zehnder-type interferometric coupler as shown in Fig. $1 \mathrm{~b}$ is given by [14]

$$
S=\cos ^{2}(\theta) \sin ^{2}\left(\phi_{1}+\phi_{2}\right)+\sin ^{2}(\theta) \sin ^{2}\left(\phi_{1}-\phi_{2}\right),
$$

where $\phi_{1}$ and $\phi_{2}$ are the half phase differences between the fundamental and first-order system modes existing in the parallel-waveguide coupler sections of each directional coupler, and $2 \theta$ $=\beta(\lambda) \Delta L$ is the relative phase delay introduced in the uncoupled section by the path length difference $\Delta L$ and the propagation constant of the waveguide mode $\beta(\lambda)$. Reference [14] specifies that in order to obtain a $3-\mathrm{dB}$ coupler with a maximally flat wavelength response, the parameters can be chosen as $\phi_{1}=\pi / 2$ (full coupler), $\phi_{2}=\pi / 4$ (3-dB coupler), and $2 \theta=2 \pi / 3$.

Single-mode silicon oxynitride $(\mathrm{SiON})$ channel waveguides with $1.8 \mu \mathrm{m}$ width and $1 \mu \mathrm{m}$ height were designed for the AWG spectrometers and the MZI. For the upper cladding 4- $\mu$ mthick silicon dioxide was used. The core and cladding refractive indices at $1.25 \mu \mathrm{m}$ wavelength were chosen as 1.54 and 1.449 , respectively, which results in an effective refractive index $\left(n_{\text {eff }}\right)$ of 1.4822. The minimum bend radius for these waveguides to have a loss $<0.01 \mathrm{~dB} / 90^{\circ}$, was calculated to be $500 \mu \mathrm{m}$. The gap in the directional couplers was chosen as $1 \mu \mathrm{m}$. The FSR and wavelength resolution $\Delta \lambda$ of the primary flat-top AWG spectrometer were chosen as $90 \mathrm{~nm}$ and $18 \mathrm{~nm}$, respectively, whereas for the secondary AWGs these values were $20.4 \mathrm{~nm}$ and $0.4 \mathrm{~nm}$, respectively. Beam propagation simulations were performed for designing and optimizing the balanced couplers. The lengths of the straight coupler sections were calculated as $L_{1}=149 \mu \mathrm{m}$ and $L_{2}=34 \mu \mathrm{m}$, and the delay length was found to be $\Delta L=0.281 \mu \mathrm{m}$, as summarized in Fig. 1b. The simulated splitting ratio between the two arms stays constant over $90 \mathrm{~nm}$ bandwidth for both couplers, as shown in Fig. 2a. In order to obtain a flat passband for all output channels, the channel spacing of the primary AWG should be equal to the FSR of the MZI, given as $\mathrm{FSR}_{\mathrm{MZI}}=\lambda_{\mathrm{c}}{ }^{2} /\left(n_{\mathrm{eff}} \times \Delta L_{\mathrm{MZI}}\right)$, where $\lambda_{\mathrm{c}}$ is the center wavelength and $\Delta L_{\mathrm{MZI}}$ is the path length difference. Therefore, assuming that the MZI and arrayed waveguides have the same effective refractive index, the MZI should have a path length difference of $\Delta L_{\mathrm{MZI}}=N \times \Delta L_{\mathrm{AWG}}$, where $N$ is the number of output channels of the AWG and $\Delta L_{\mathrm{AWG}}$ is the constant length increment between adjacent arrayed waveguides. For the primary AWG the values of $N, \Delta L_{\mathrm{AWG}}$, and grating order were $5,11.79$ $\mu \mathrm{m}$, and 14, respectively. The excess loss decreases monotonically as the gap between waveguides in the second 3-dB balanced coupler of the MZI configuration becomes narrower, because the excess loss originates from the field mismatch between an even mode in the second 3-dB balanced coupler and a fundamental mode in the output waveguide at the center wavelength. Therefore, the gap between waveguides at the 3-dB balanced coupler and the star coupler interface was arranged to be $0.8 \mu \mathrm{m}$ by replacing its bent output waveguides by a small extension of the straight section $L_{2}$ from $34 \mu \mathrm{m}$ to $40 \mu \mathrm{m}$. The performance of the reconfigured coupler is similar to the original one. The proposed AWGs were fabricated using plasma-enhanced chemical vapor deposition, conventional photolithographic patterning, and reactive ion etching in the given order. The primary and the secondary AWGs were on the same chip with an overall size of $4.5 \times 1.5 \mathrm{~cm}^{2}$.

Due to the energy distribution among the diffraction orders in a cyclic AWG, i.e. FSR $=N$ $\times \Delta \lambda$, the peripheral output waveguides suffer almost $3 \mathrm{~dB}$ more loss than the center waveguide (i.e. loss non-uniformity) [1], thereby in a cascaded system the loss non-uniformity of the secondary AWGs will be at maximum equal to the $3 \mathrm{~dB}$ of a cyclic AWG. For the primary AWG design, the loss around the crossing points with the adjacent waveguides was reduced by designing the passband width in such a way that the adjacent channels overlap 
around the 3-dB down point in order to have a uniform intensity distribution for the complete cascaded system. In principle, using a non-cyclic AWG with a much larger FSR could result in a loss non-uniformity smaller than $3 \mathrm{~dB}$, however, at the expense of a larger device size.
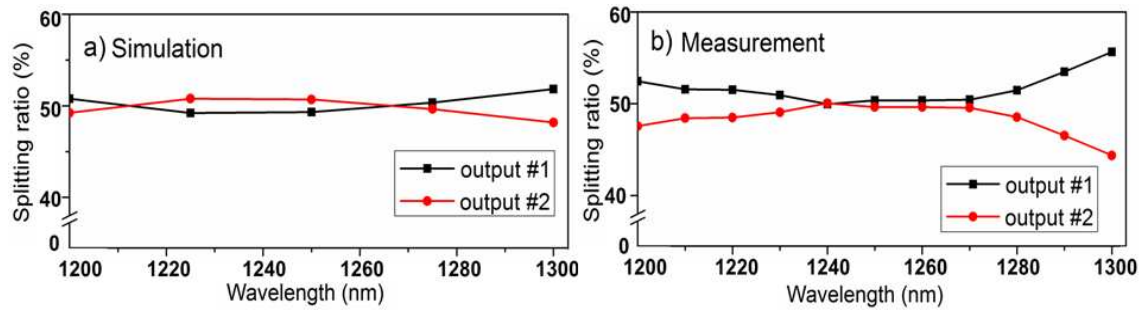

Fig. 2. a) Simulation and b) measurement result of the $3-\mathrm{dB}$ balanced coupler. The wavelength dependence of the coupler increases at longer wavelengths due to processing fluctuations.

\section{Measurements}

The measurements on both the individual 3-dB balanced couplers and the cascaded AWG system were performed with the same measurement set-up shown in Fig. 2.10, by coupling TE-polarized light from a broadband source (Fianium SC450) alternatively into the 3-dB balanced coupler or the synchronized AWG with a single-mode polarization-maintaining fiber. The output signal was sent through a single-mode fiber to an optical spectrum analyzer (iHR 550, Horiba Jobin Yvon). The transmission spectra measured at the output waveguides of the different investigated structures were normalized with respect to the transmission of a straight channel waveguide. The measured spectra of the 3-dB balanced coupler are displayed in Fig. 2b. Due to fluctuations in the fabrication process, the core refractive index turned out to be slightly higher than intended, which led to a degradation of the performance of the 3-dB balanced coupler, especially at longer wavelengths. The new core effective refractive index for TE was calculated as 1.548 from the measured central wavelength shift of the MZIsynchronized AWG. A simulation with a beam propagation method using the increased core refractive index showed a similar performance decrease. In addition, at wavelengths where the splitting ratio is not exactly $3 \mathrm{~dB}$, the coupler causes an additional phase shift between its outputs, which may cause reduced synchronized-AWG performance in terms of passband symmetry, passband ripple, and excess loss at the outer channels.

The MZI-synchronized AWG was characterized using the aforementioned measurement procedure. The measured spectra are displayed in Fig. 3a. A 0.5-dB bandwidth of $12 \mathrm{~nm}$ and a central excess loss value of $1 \mathrm{~dB}$ were measured. A central wavelength shift of $7 \mathrm{~nm}$ with respect to the designed value was observed, corresponding to the same effective refractive index of the core of 1.548 that explains the measured performance of the 3-dB balanced coupler. At longer wavelengths the performance of the synchronized AWG decreased due to degraded performance of the $3-\mathrm{dB}$ couplers, which was verified by the BPM simulations shown in Fig. 3a by dashed lines for the center and the $5^{\text {th }}$ output channels.

In order to overcome fabrication-related performance degradation of the MZIsynchronized AWG, chromium electrical heaters were placed on both arms of the MZI. These heaters control the phase delay between the modes in the two waveguides and, thus, the field distribution at their junction with the AWG input free-propagation region, and thereby also the AWG passband shape. Figure $3 \mathrm{~b}$ shows the transmission response of one of the output waveguides of the flat-top AWG with and without thermal tuning. The asymmetrical passband shape due to the fabrication imperfections was significantly improved by applying $0.5 \mathrm{~W}$ to the bottom arm of the MZI.

The cascaded AWG configuration is shown in Fig. 1c. This device was characterized using the same procedure as described before. Several of the outer and central output waveguides of each secondary AWG were measured, as shown in Fig. 4. The overall response has an envelope function which overlaps very well with the transmission spectra of the MZIsynchronized primary AWG, as indicated by the dashed line in Fig. 4a. A central excess-loss 
value of $4.5 \mathrm{~dB}(1 \mathrm{~dB}$ from the primary AWG and $3.5 \mathrm{~dB}$ from the secondary AWG) and a non-adjacent crosstalk value of $30 \mathrm{~dB}$ were obtained. A close-up of the transmission measurement results of the $4^{\text {th }}$ secondary AWG is provided in Fig. $4 \mathrm{~b}$. The loss values at the spectral crossing points of the adjacent passbands of the primary AWG increased from $3 \mathrm{~dB}$ to $6 \mathrm{~dB}$ towards longer wavelengths, as seen in Fig. 3a, which can be compensated for in future designs by arranging the passband width of the primary AWG.
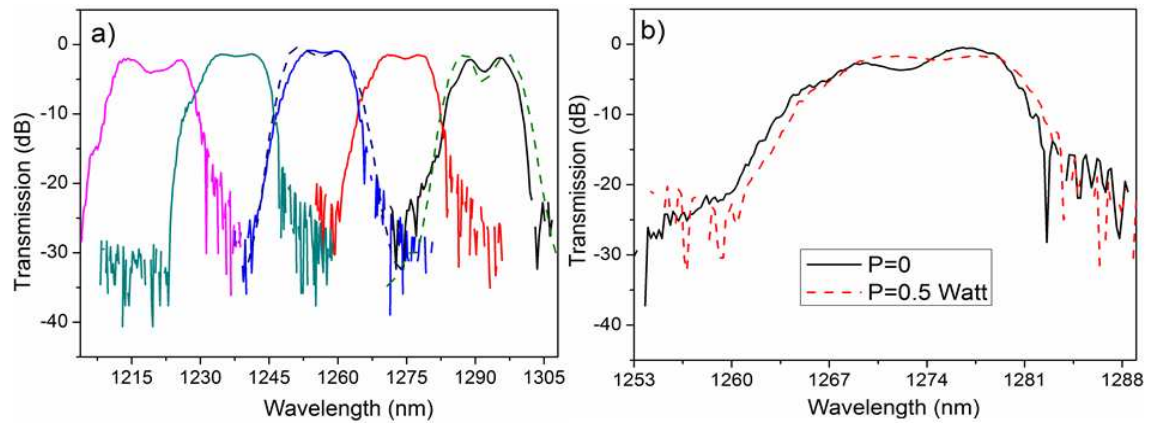

Fig. 3. a) MZI-synchronized AWG spectrum, exhibiting a $0.5-\mathrm{dB}$-bandwidth of $12 \mathrm{~nm}$ and 1 $\mathrm{dB}$ excess loss at the central channel. The dashed lines are the simulated transmission spectra of the center and the $5^{\text {th }}$ output channels in case of the non-ideal balanced coupler given in Fig. $2 \mathrm{~b}$.

b) Thermal tuning effect on the transmission spectrum of one of the output channels of the MZI-synchronized AWG; black solid line: heater turned off, red dashed line: heater turned on.
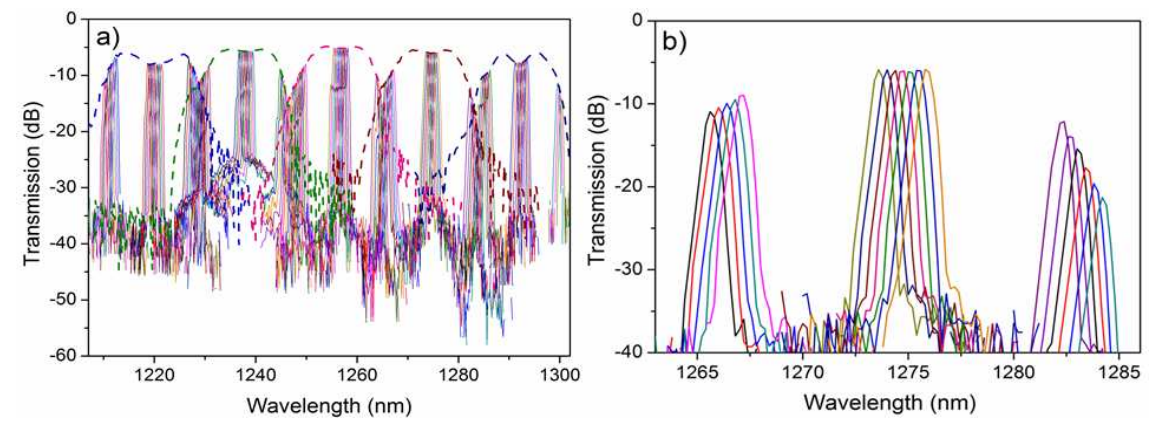

Fig. 4. a) Measurement result of the cascaded AWG system with MZI-synchronized, flat-top primary AWG and five $1 \times 51$ secondary AWGs. The dashed line is the transmission response of the MZI-synchronized primary AWG, which acts as an envelope for the secondary AWGs. b) Close-up of the $4^{\text {th }}$ secondary AWG transmission results.

\section{Conclusions}

A new pass-band flattening method was demonstrated by introducing 3-dB balanced couplers to a MZI-synchronized AWG configuration over a broad spectral range. A 0.5 -dB bandwidth of $12 \mathrm{~nm}$ and a central excess loss value of $1 \mathrm{~dB}$ were measured. Electrical heaters were placed on both arms of the MZI in order to compensate the fabrication-related performance degradations. As a proof-of-concept a cascaded AWG system was demonstrated, which consists of the low-loss flat-top AWG as a primary filter and five $1 \times 51$ AWGs with a $0.4-\mathrm{nm}$ channel spacing as secondary filters. Such low-loss, broad-spectral-range AWGs are very desirable for high-density cascaded multiplexer/demultiplexer systems.

\section{Acknowledgments}

This work was supported by funding through the Smartmix "Memphis" program of the Dutch Ministry of Economic Affairs and the Netherlands Ministry of Education, Culture and Science. The authors thank Xaveer Leijtens for fruitful discussions and Anton Hollink for technical support. 